Results LOS reduction efforts resulted in days saved in nonCOVID patients, but the negative savings from COVID patients resulted in a net loss of opportunity days when all patients were bundled together (figure 3). Calculations for opportunity days saved were modified to account for the decrease of inpatient volumes and subsequent analysis of the

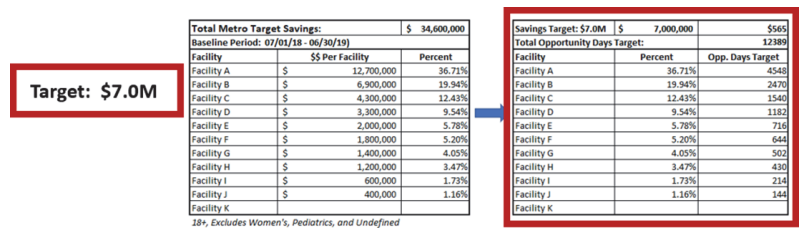

Abstract 3 Figure 1

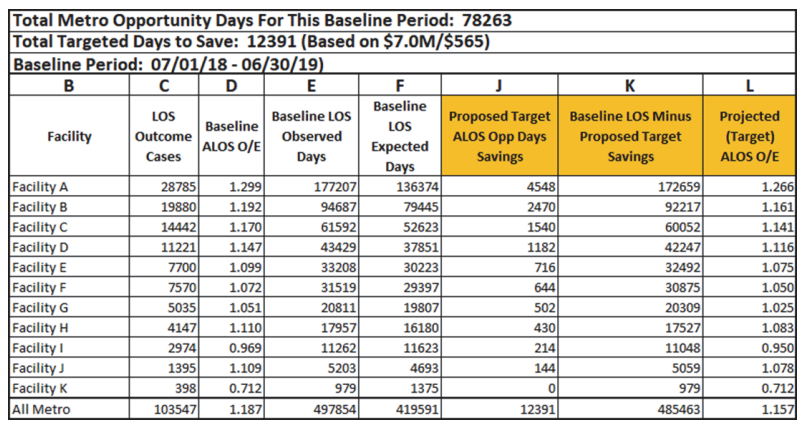

Abstract 3 Figure 2

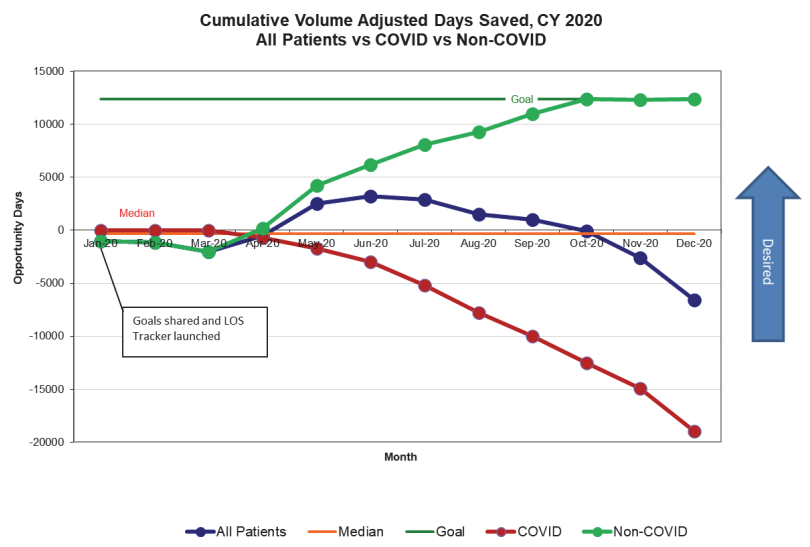

Abstract 3 Figure 3

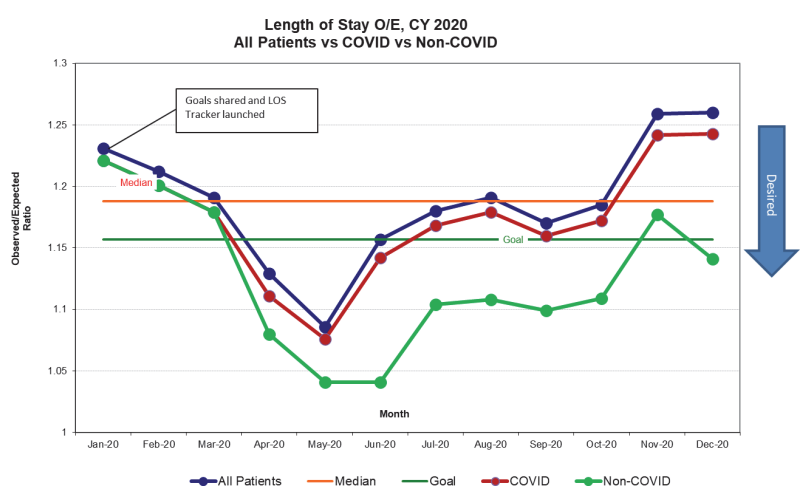

Abstract 3 Figure 4 data included stratification for the COVID vs. non-COVID population.

Conclusions The model described for combining a financial goal by facility with opportunity days enables the setting of LOS targets for healthcare systems. Further, the model supports tracking progress to targets, including the ability to compare specific patient types, e.g., COVID-19 Positive vs. COVID-19 Negative (figure 4).

\section{RIGHT CARE AT THE RIGHT TIME: A CARE MODEL REDESIGN QUALITY IMPROVEMENT PROJECT}

William Bekker, Caitlin Garrett, Heather Gleason, Kathryn Hoehn, Katie Iommazzo, Karen Lane, Benji Mathews, Hilary Radtke, Sara Spilseth, Julie Weegman. Regions Hospital

\subsection{6/bmjoq-2021-|HI.4}

Background Over the last several years, there have been focused efforts to continually improve communication amongst the care team while maintaining patient and family centered care, progressing the patient through their care as clinically indicated, and ensuring timely and efficient discharge planning. Despite these efforts, there was a lack of reliability in the care model which created gaps amongst care team members (inclusive of the patient) in the knowledge of the plan of care.

Objectives Care Model redesign is an approach aimed to decrease overall length of stay, improves workflow efficiencies that prioritize quality and timely care, and set a "gold standard" for hospital patient care. The goal of the inpatient care model was to improve both patient and staff satisfaction, while ensuring processes that support patient throughput and overall access to care.

Methods The Model for Improvement, best practice review, adaptive change management, human centered design tools, and PDSA cycles were utilized within the project. We created a new care model with structured connections and tools to support discussions. We engaged front line staff through a design session and optimized current technology to merge segmented work into one cohesive approach.

The new care model was initially piloted, evaluated, and spread to eight units across the hospital. The team leveraged a multidisciplinary coaching model, and consistent leadership presence to ensure engagement of all team members.

Results The data highlights reduced variation by creating a more reliable model to replicate. This resulted in a $21 \%$ rate of improvement, and we have exceeded the organization's goal of $20 \%$ of discharges by 11:00am (figure 1). The team identified process, outcome, and balancing metrics for this project that were closely monitored and reviewed (figure 2).

Conclusions There is a continued commitment to follow the new process 7 days a week, identify opportunities and

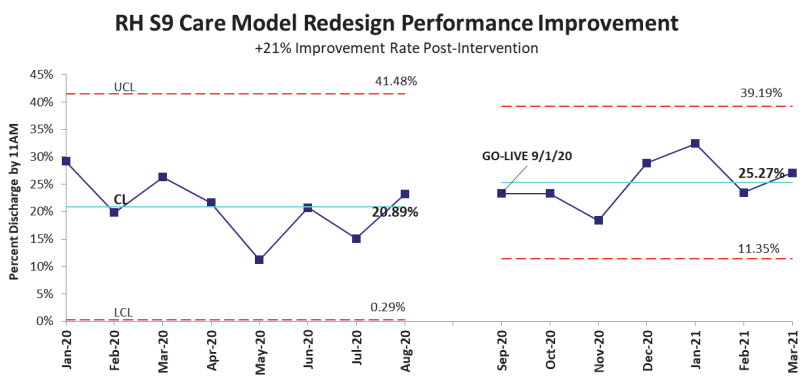

Abstract 4 Figure 1 


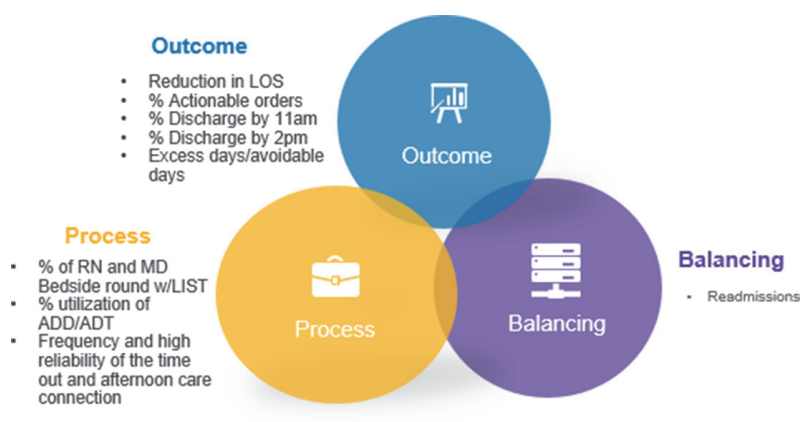

\section{Abstract 4 Figure 2}

problem solve a patient's plan of care as a team, and maintain high reliability. Communication and coordination amongst care teams and complex discharge planning has improved. Team members have demonstrated workflow efficiencies by being proximate to patients and each other.

\section{RECOVERING FROM COVID - IMPROVING OPERATING ROOM CAPACITY USING ADAPTIVE CLINICAL MANAGEMENT}

Mark Cain, Nicolas Fernandez, Daniel Low, Paul Merguerian. University of Washington, Seattle Children's Hospital

\section{$10.1136 / \mathrm{bmjoq}-2021-\mid \mathrm{HI} .5$}

Background U.S. hospitals will lose more than \$53B revenue due to COVID-19 in 2021. The operating room (OR) is the revenue engine of the hospital, 60\% of their operating margin comes from perioperative services. Optimizing capacity is essential to financial recovery.

Objectives The aim of this project was to use Adaptive Clinical Management to increase operating room (OR) capacity by improving efficiency (figure 1).

Methods The distinct phases of OR care were mapped. Surgery Prep Time was defined as the interval between anesthesia readiness and surgical procedure start time. Tasks include moving the patient to the OR table, positioning, prepping the surgical field, and performing safety time-outs. Electronic health records (EHRs) routinely record anesthesia readiness and procedure start time stamps. EHR data was extracted and analyzed by SPC charts using AdaptX (Seattle, WA). Inter-surgeon performance variation was quantified using funnel plots. Interviews with the best performing surgeons informed standard work protocols which were implemented by a clinical champion, then by the wider group. Daily clinical performance data updates enabled surgeons to rapidly adapt their workflows to improve efficiency.

Results Baseline surgical prep time was 13.7 minutes (figure 2). Surgeons' variation in prep times were quantified using a funnel plot (figure 3), two surgeons identified with shorter prep times, one outside the 3-sigma lower control limit (special cause variation). Commonalities in their practices were

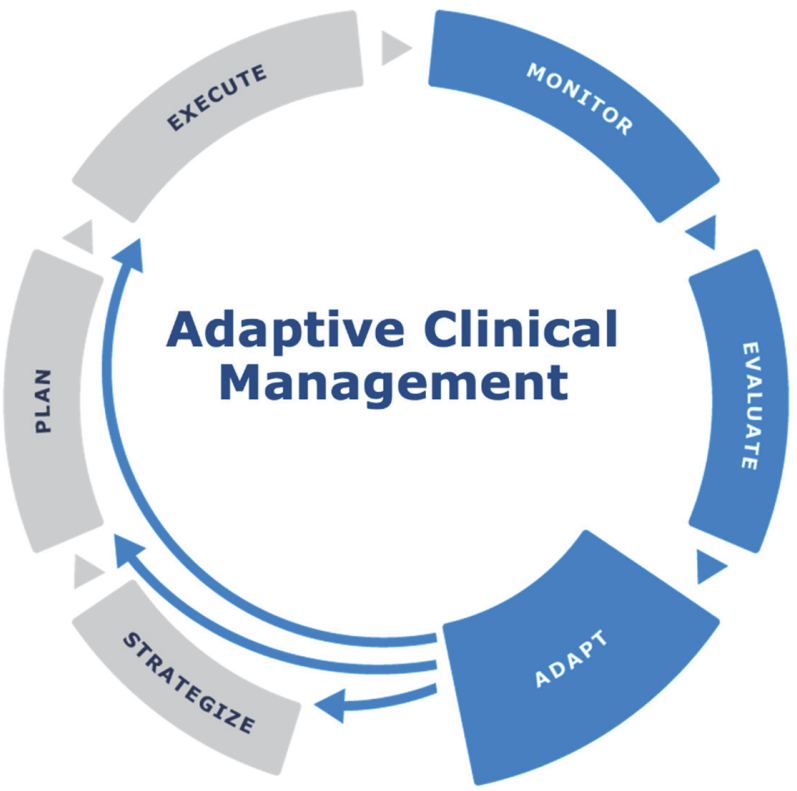

Abstract 5 Figure 1 Adaptive clinical management. A framework for clinicians to dynamically use real-world data to drive improvement in both clinical and operational processes

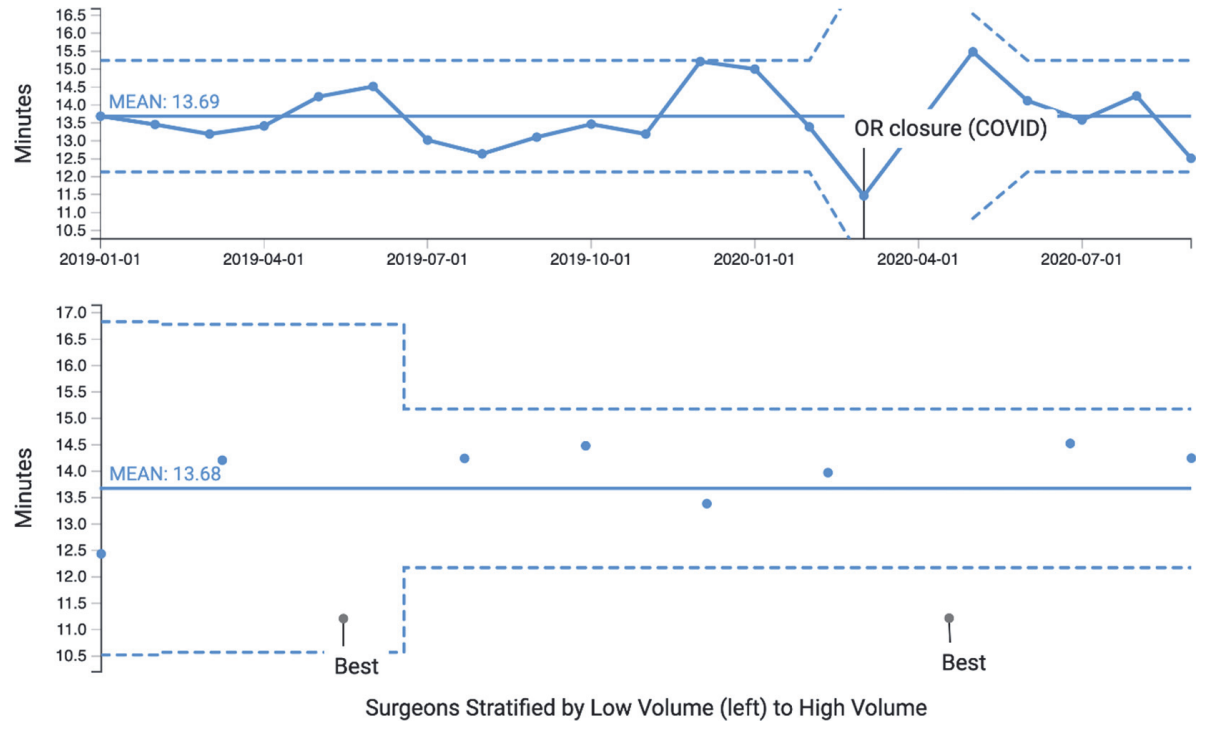

Abstract 5 Figure 2 Surgical prep time - historical baseline system performance (above). Funnel plot -Stratifies individual surgeon's performance and identifies best practice (below) 